\title{
Application of laser Compton photon beam to nondestructive tests
}

-A spin-off technology from nuclear physics -

\author{
Hiroyuki TOYOKAWA
}

[Translation from Synthesiology, Vol.8, No.2, p.89-96 (2015)]

\begin{abstract}
Laser Compton photon beams generated by a high-energy electron storage ring have energy in the gamma-ray range. X-ray radiography for industrial products using the laser Compton photon beam is expected to show good spatial and density resolutions, because of its monochromaticity and good beam property. A radiography and computerized tomography system was built using AIST's TERAS electron storage ring. The performance of the system was examined. A summary of the development process is outlined to figure out the general methodology for translational research.
\end{abstract}

Keywords : Electron accelerator, laser, radiation, radiography, nondestructive inspection

\section{Introduction}

Translational research is a process in which scientific knowledge for basic research is translated into practical application for the benefit of society. While we attain the objectives of research, we take several issues into account, such as schedule, quality of research output, funding, cost efficiency for investment, objectives of the organization, and the voice of society. Technical level might be lowered if we easily compromised to produce outcomes in a short time, or we may become isolated from society if we keep pursuing our own way.

The objective of this paper is to show how a basic research study in an electron accelerator translated into industrial technology. The translation process is not to improve the device performance or produce champion data, but is to bridge research results to industry in an appropriate form.

The present research consists of the following scenario:

(1) Development and compilation of elemental technologies.

(2) Open to users: Device open to users to estimate potential users by listening to the users' voices.

(3) Investigation of plan: To investigate the research plan by objectively understanding the users' voices on whether the obtained results were as expected initially, what was overlooked if the results were not as expected, or whether there were better ways.

(4) Modification of plan: To organize the main points of future devices and technologies. To determine whether the research will be continued by considering the costeffectiveness and the situation of the organization. To act quickly according to the decision made.

This paper consists of four chapters. In chapter 2, technical issues will be discussed. Chapter 3 discusses the process of selection and integration of technologies for bridging, in correspondence to $(1) \sim(3)$ listed above. Chapter 4 reflects on the process of selection and integration of the elemental technologies in correspondence to (4), and discusses the efforts spent to achieve the goals and results.

\section{Technical issues about laser Compton scattering}

There are attractive technologies in nuclear physics and elementary particle physics especially in radiation measurement technologies. They are highly sophisticated, and can be applied to industrial technology with some modifications. We applied a method for measuring cross-sections of atoms and nuclear reactions to nondestructive testing via industrial radiography.

Industrial radiography is an important technique in improving reliability of industrial products such as automobiles, aircraft, rockets, sintered materials, cast products, and electronic substrates. ${ }^{[1]}$ A high-energy X-ray computerized tomography (CT) system using an electron accelerator that is capable of radiographing a whole engine block at spatial resolution of $2 \sim 3 \mathrm{~mm}$ has been developed. In infrastructure diagnosis, the technology to inspect roadbeds and bridges on site using a portable electron accelerator has been developed recently. ${ }^{[2]}$ A nondestructive and highly precise method for visualizing reinforcing steel-bars and cracks in concrete structure is strongly demanded in our society.

Research Institute of Instrumentation Frontier, AIST Tsukuba Central 2, 1-1-1 Umezono, Tsukuba 305-8568, Japan * E-mail : h.toyokawa@aist.go.jp

Original manuscript received September 15, 2014, Revisions received December 27, 2014, Accepted January 19, 2015 
The word "photon" is the name for quantized electromagnetic waves, and in this paper, it is used to mean X-rays or gamma rays. Radiography is a photographic method using ionizing radiations, and this includes so-called roentgen photography and cross-sectional photography using X-ray CT.

In this research, laser Compton scattering (LCS) ${ }^{[3]}$ technology using an electron storage ring is used. An electron storage ring is a circular electron accelerator, in which electrons are enclosed in a donut-shaped ultra-high vacuum chamber by magnetic fields, and are accelerated using electric fields. We used an electron storage ring with a diameter of $10 \mathrm{~m}$ and a circumference of about $30 \mathrm{~m}$, which is a medium- to smallscale device.

LCS is one of the methods to generate high-energy photons using an electron accelerator. When laser beams are irradiated onto high-energy electron beams, laser photons are scattered with the electrons, receiving part of the electron energy through Compton scattering, and become X-rays and gamma-rays. Compton scattering is the collision of photons and electrons, and the photon energy before and after scattering, scattering angle in the laboratory frame, and relationship with electron energy in LCS are expressed in Equation 1.

$$
E_{\gamma}=\frac{E_{0}\left(1-\beta \cos \theta_{1}\right)}{1-\beta \cos \theta_{2}+E_{0} \frac{1-\cos \left(\theta_{2}-\theta_{1}\right)}{E_{e}}}
$$

The kinematics is shown in Fig. 1. The LCS photon research started in 1985 at the Electrotechnical Laboratory (later became part of AIST), and the photon beam source at the photon range of $1 \sim 40 \mathrm{MeV}$ was available for scientific studies since 1990 s. $^{[4][5]}$ Since high-energy and highly-oriented photon beams can be obtained, it has been used in much research such as nuclear physics studies, measurement of
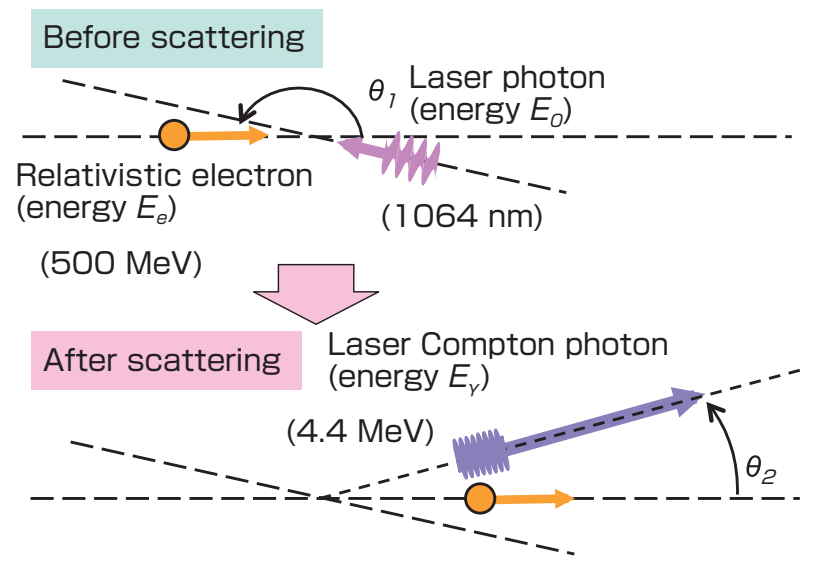

(Laboratory frame coordinate system)

Figures in ( ) are sample values used in experiment

Fig. 1 Kinematics of laser Compton scattering response function for radiation detectors, measurement of cross sections of photonuclear reactions, and measurement of absorption cross sections of atoms.

\section{Process of the selection and integration of technologies}

(1) Development and sophistication of the elemental technologies

For industrial radiography, a spatial resolution of $1 \mathrm{~mm}$ or less is necessary to detect foreign materials. To detect and evaluate the air bubbles in resin, the resolution of approximately $10^{-2} \mathrm{~cm}^{-1}$ is necessary as the absolute value of linear attenuation coefficient. ${ }^{[6]}$ A photon flux that passes through a substance exponentially attenuates against the distance travelled, which is characterized by the linear attenuation coefficient that has the dimension of inverse length.

In X-ray CT systems, the attenuation of photon intensity as a function of the penetration depth is assumed to be exponential. However, as shown in Fig.2, the linear attenuation coefficient is a function of the substance and the photon energy. Because the conventional X-ray CT system uses white-colored X-rays that contain various wavelengths (energy), the CT image is the result of the convolution of various X-rays with different linear attenuation coefficients. As a result, many artifacts appear in the CT images. In medical CT, all substances can be considered more or less equivalent to water since the subject measured is mainly living bodies. So, it is possible to correct the artifacts by using a water phantom in medical CT.

Because various substances of wide dynamic range of linear attenuation coefficients will be considered for industrial CT, it is difficult to correct artifacts and to evaluate precisely the density and material distribution. A CT system using monochromatic photons with sufficient transmissivity can

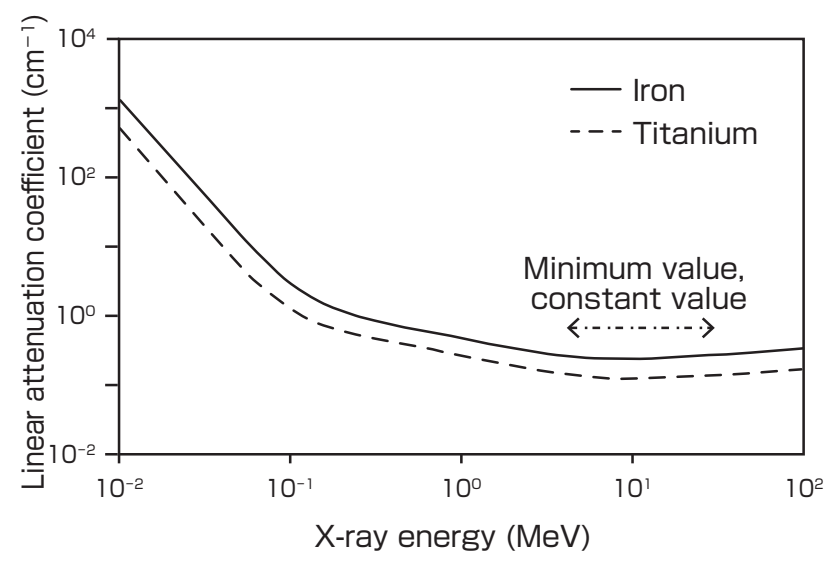

Fig. 2 Linear attenuation coefficients of iron and titanium at $10 \mathrm{keV} \sim 100 \mathrm{MeV}^{[6]}$ 
overcome this difficulty.

As shown in Fig. 2, the linear attenuation coefficients for industrial materials remain almost constant for photon energy of $5 \sim 20 \mathrm{MeV}$. For example, the linear attenuation coefficient for iron shows a shift of $0.6 \%$ at photon energy of $10 \pm 3$ $\mathrm{MeV}$. This value is equivalent to the energy spread of several $\mathrm{eV}$ per $1 \mathrm{keV}$ of photons. That is, the CT measurement using photons at $\mathrm{MeV}$ range of little over $10 \%$ becomes equivalent to the photon CT measurement with energy spread of $1 \%$ or less at $\mathrm{keV}$ range, and therefore it can be regarded as using monochromatic photons. The linear attenuation coefficient becomes the minimum in the $\mathrm{MeV}$ range for many substances. Therefore, photons readily pass through substances in the $\mathrm{MeV}$ range. That is, the photon beams at $\mathrm{MeV}$ range are the optimal tool for testing thick samples at high density resolution.

High spatial resolution, high density resolution, and high time resolution (short measurement time) are required for a good industrial radiography system. To achieve these concurrently, it is necessary to scan samples with a pencil-like thin beam at a small pitch, or with a cone-like beam and an X-ray camera with a small pixel with high detection efficiency.

Models that realize these properties are shown in Fig. 3. The diagram in (A) is the CT method where scanning is
(A)

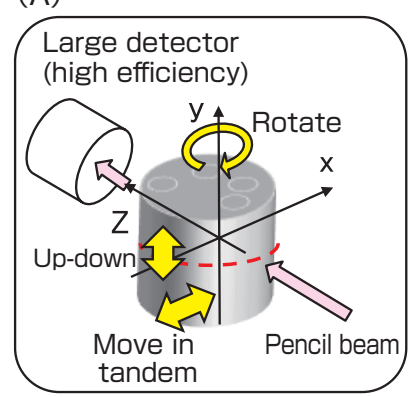

First-generation CT

(In tandem $\leftrightarrow$ rotate $\leftrightarrow$ up-down)

- High detection efficiency

- Pixel is determined by photon

beam diameter

(High image quality)
(B)

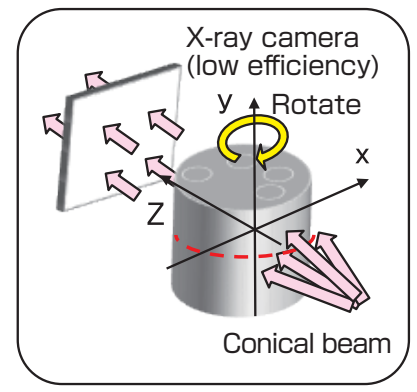

Third-generation CT

(rotation only)

- Low detection efficiency

- Pixel is determined by size of camera element

(Image blur is large)
Fig. 3 Explanation of CT generations. (A) First-generation and $(B)$ third-generation. done using a thin beam, which is called the first-generation $\mathrm{CT}$. The sample is moved perpendicularly to the beam axis, moved up and down, and rotated 360 degrees to measure the transmission image. The diagram in (B) shows the thirdgeneration $\mathrm{CT}$ method where the transmission image is obtained without moving the sample by using the cone-beam and the X-ray camera placed behind the sample. While the third-generation CT system has an excellent time resolution, the spatial resolution cannot be improved better than a few $\mathrm{mm}$, because of the scattered X-rays within the camera.

Because the technical goal of the present research is to develop a prototype industrial CT system using $\mathrm{MeV}$ photons, and to demonstrate high numerical performance for density and spatial resolutions with fine CT images, we built a first-generation CT system in this study. The outline of the first-generation CT system developed in this research is shown in Fig. 4.

\section{(2) Open user research}

We have successfully developed the elemental technologies and the CT system. The next step was to enhance the utility of the system by supplying the CT system for open use, and to continue discussions with the users on improvement points and requirements. The system was actually released to open user experiments to hear their voices.

We conducted joint research with companies in the automobile and electricity industries in this research phase, and found their demands were to have spatial resolution of at least $1 \mathrm{~mm}$ or less, and to detect inhomogeneity in density distribution of $1 \%$ or less. The measurement results of transmission images of industrial products using the present radiography system are shown in Fig. 5 (left). We succeeded in demonstrating the principles but failed to obtain satisfactory images in the photography tests for metal bolts that were conducted in the early stages of this R\&D.

Various improvements were conducted to enhance the performance. ${ }^{[7]}$ For example, we improved the alignment precision of the collimator, the spatial-and-temporal collision precision of the laser and electron beams, intensity and stability of the LCS photons, and so on. We made an electron

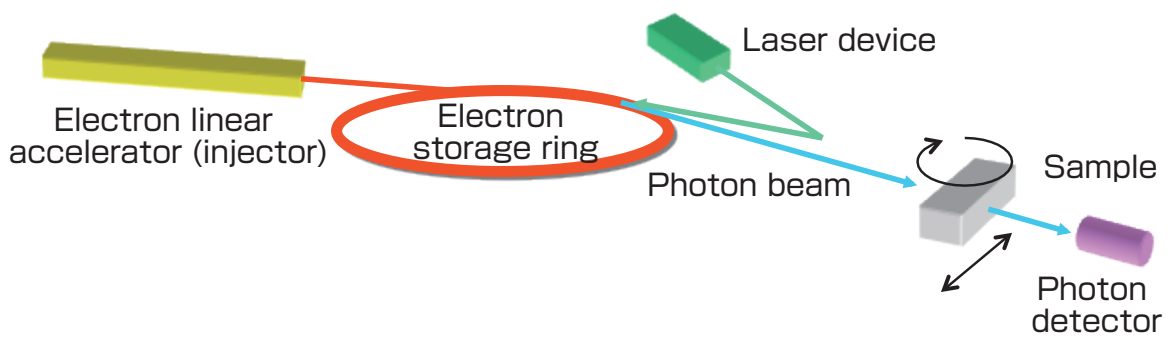

Fig. 4 LCS photon CT system using the electron accelerator 
accelerator system that could be operated by one person, so that users could tune the LCS photon energy and intensity online.

The image quality improved, and the CT image of spatial resolution of $1 \mathrm{~mm}$ or less was obtained. ${ }^{[8]}$ We also obtained highly precise CT images (Fig. 6). ${ }^{[9]}$ The CT numbers were accurately measured for various substances, and they were proportional to the linear attenuation coefficients in a wide dynamic range. We succeeded in achieving density resolution of $1 \%$ or less (Fig. 7). ${ }^{[10]}$ We were able to demonstrate its effectiveness as a measurement technology for infrastructure diagnosis such as for cracks of $0.2 \mathrm{~mm}$ width in concrete and $\mathrm{CT}$ of cross sections of reinforced steel concrete with $50 \mathrm{~cm}$ thickness. ${ }^{[11]}$ Figure 8 shows how the elements are integrated to make a system.

\section{(3) Verification of the research results}

As the automation of the device progressed, it became possible for the users themselves to conduct adjustment operation of the accelerator and to generate the LCS photon beam. This started to produce results in the CT research for open use. Joint research was conducted with companies and universities for many years. In this period, evaluations and improvements of the spatial and density resolutions were conducted, and we were able to achieve the performance that was initially set as our goal.

The users gradually voiced requests for the development of small devices that can be installed in their own factories. To accomplish this, downsizing and cost reduction of the accelerator are mandatory.

We need an electron storage ring or a continuous-beam electron accelerator of a few hundred $\mathrm{MeV}$ for the present method. Downsizing the CT system is almost equivalent to downsizing the electron accelerator. However, since the accelerator energy is proportional to the system size, it is difficult simply to decrease the size of each device. More development is needed such as the development of materials and a surface treatment technology that can resist high electric fields of several $100 \mathrm{MV} / \mathrm{m}$ or more; a machining technology of nanometer precision and technology; a
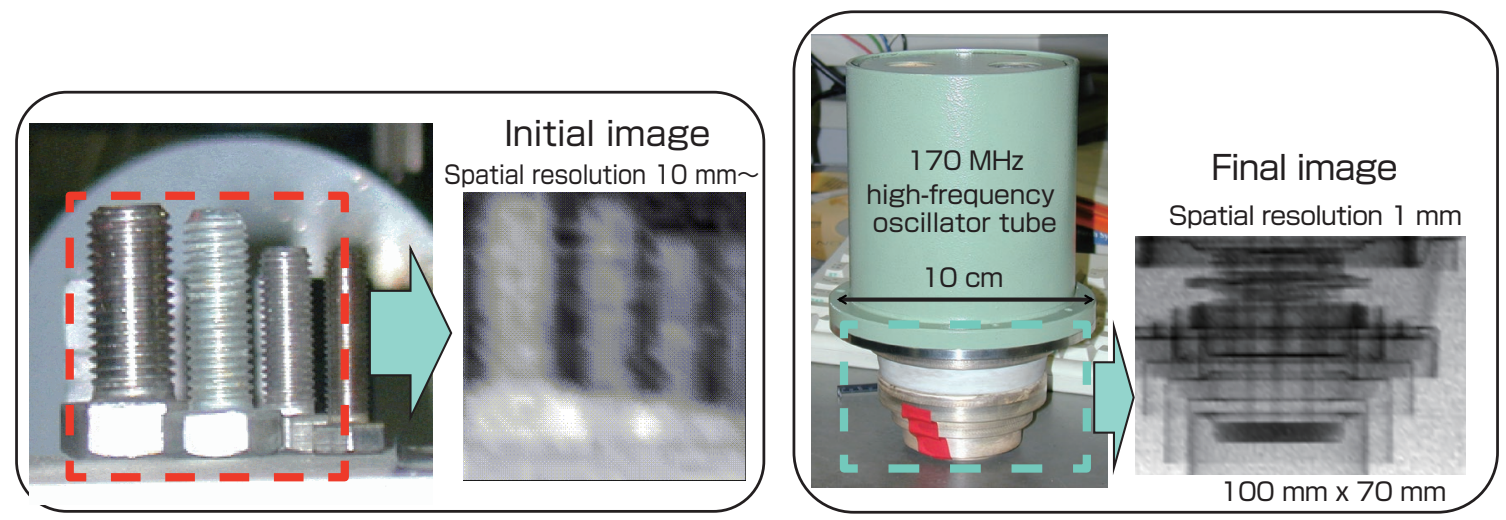

Fig. 5 Transmission radiography using LCS photons

Left: Stainless bolts were photographed using $10 \mathrm{MeV}$ LCS photons (black-white are reversed). Right: Electrode part of highfrequency oscillator tube was photographed using $10 \mathrm{MeV}$ LCS photons (spatial resolution $1 \mathrm{~mm}$; part of the diagram was cited from Reference [8]).
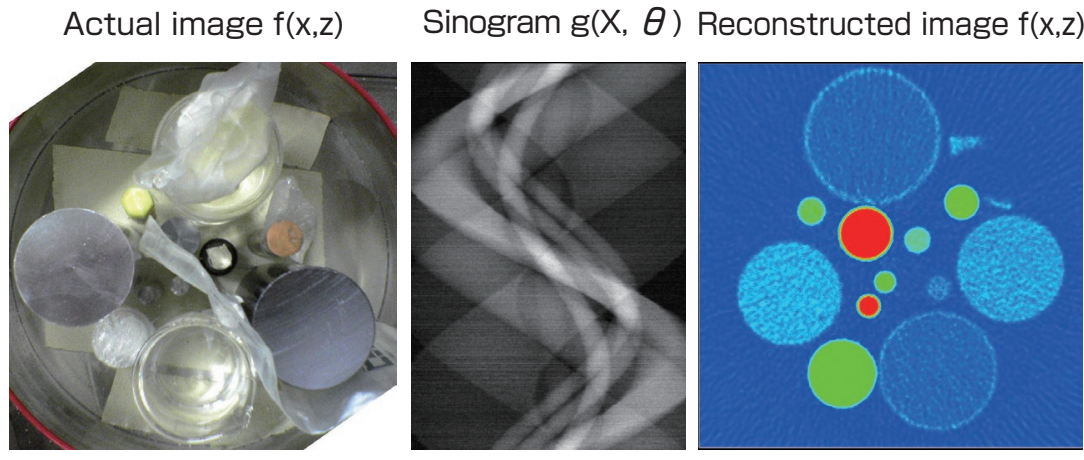

Fig. 6 (Left) Sample photograph, (center) sinogram, and (right) CT image ${ }^{[9]}$ The samples include water in a beaker and rods of various metals such as silicon, aluminum, and tungsten. Blue to green represent the low density ranges, while yellow to red show the high density ranges. 
magnet that can achieve highly precise magnetic field; and improvement in bunch charge density and management of severe space charge effect. Downsizing and weight reduction are also necessary for all parts being used. It is difficult to achieve full performance equivalent to that of a large accelerator system with a small accelerator system. The best way is to develop an electron accelerator of specific performance for specific usage, with a moderate cost.

Now, the usefulness and the problem of an industrial highenergy photon CT system became clear. Unlike the medical X-ray CT system, various materials are the subjects of measurement in industrial radiography. So, it is important to accurately measure the shape, outer and inner sizes, and the density distribution of industrial products using a highenergy X-ray CT system. A research project to evaluate the precision and reliability of the measurement of the inner structure of industrial products obtained with $\mathrm{CT}$ images compared with other methods, such as a three-dimensional coordinate measuring machine, is being conducted, as one of the important translational research projects for industry. ${ }^{[12]}$

\section{Future developments}

At present, the present research has entered the phase of investigating downsizing and weight reduction of the electron accelerator for a specific purpose. A $0.9 \mathrm{MeV}$ portable electron accelerator system for infrastructure measurement is being developed. We use a coniferous carbon nanostructure (CCNS) material $^{[13]}$ as an electron emitter for the electron gun to minimize the system size and weight. We started up a project to build a radiography system that observes the internal part using backward scattering of X-rays, and to apply this system to nondestructive tests for infrastructures. ${ }^{[14]}$

Although it may be a repetition of the first chapter, the scenario of the present research is as follows.

(1) Development and compilation of elemental technologies
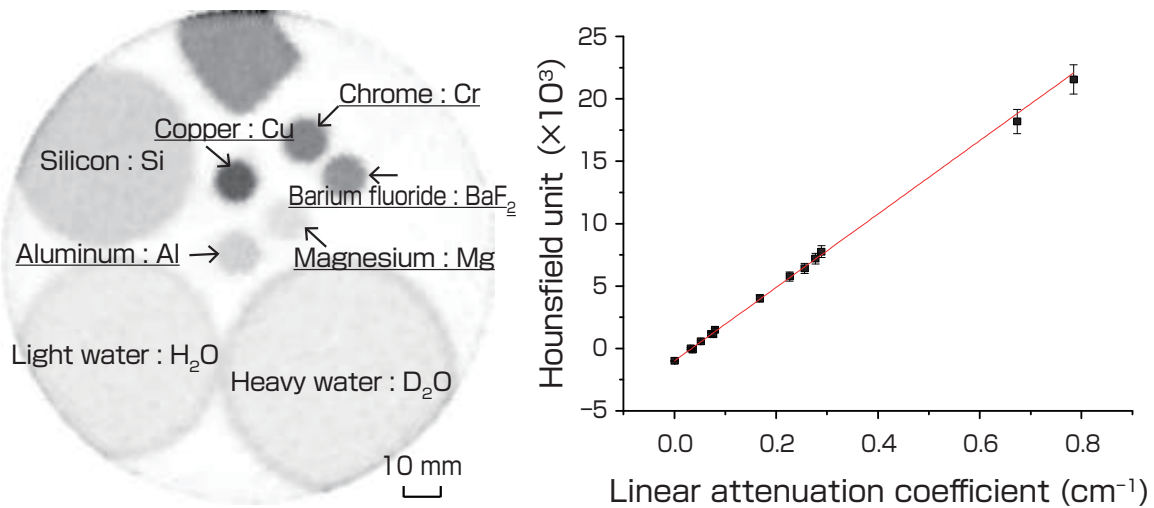

Fig. 7 (Left) Plot of the linear attenuation coefficients of various materials and the CT values expressed by HU. ${ }^{[10]}$ The symbols in the diagram represent the substances, e.g. $\mathrm{D}_{2} \mathrm{O}=$ heavy water.

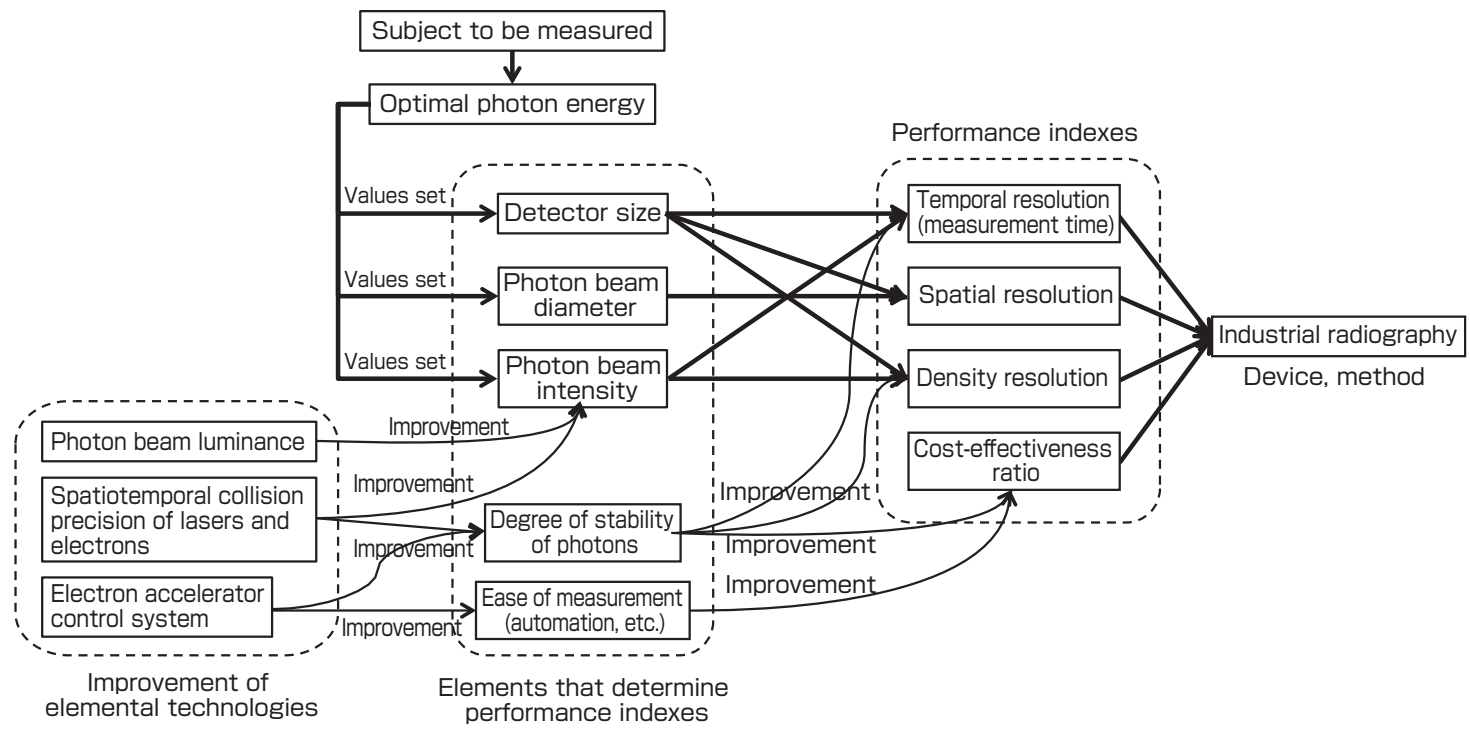

Fig. 8 Improvement of elemental technologies, elements that determine the performance index, and relationship of the sophistication of devices and methods 
(2) Open to users

(3) Investigation of plan

(4) Modification of plan

By conducting the research according to the above flow, we believe translational research can be conducted efficiently. Particularly, the user response of (2) is a job that requires concentration and physical energy, and if one stays too long at this phase or concentrate research resources here, the main objective turns into providing stable supply of devices, and we may miss the timing to move on to (3). Phase (2) is a passing point, and it is necessary to make a managerial decision to move forcibly to (3). Since the bridging research involves the researchers listening directly to the social demands, strong consciousness and motivation are generated among the researchers. If translational research is conducted in a bottom-up style, there is danger that the research resource of the organization becomes fragmented and the governance may decline. To promote this research, it is necessary to also conduct revisions to strengthen the organization.

\section{References}

[1] M. Fujii: X-ray micro-computed tomography, Journal of the Japanese Society for Non-Destructive Inspection, 54 (5), 228-232 (2005) (in Japanese).

[2] M. Uesaka, T. Natsui, K. Lee, K. Dobashi, T. Yamamoto, T. Fujiwara, H. Zhu, K. Demachi, E. Tanabe, M. Yamamoto, N. Nakamura, J. Kusano, T. Higo, S. Fukuda, M. Yoshida and S. Matsumoto: $950 \mathrm{keV}, 3.95 \mathrm{MeV}$ and $6 \mathrm{MeV}$ X-band linacs for nondestructive evaluation and medicine, Nucl. Instrum. and Methods in Phys. Res. A, 657 (1), 82-87 (2011).

[3] R. H. Milburn: Electron scattering by an intense polarized photon field, Phys. Rev. Lett., 10 (3), 75-77 (1963).

[4] T. Yamazaki, T. Noguchi, S. Sugiyama, T. Mikado, M. Chiwaki and T. Tomimasu: Generation of quasimonochromatic photon beams from Compton backscattered laser light at ETL electron storage ring, IEEE Trans. Nucl. Sci. NS, 32 (5II), 3406-3408 (1985).

[5] T. Tomimasu, T. Noguchi, S. Sugiyama, T. Yamazaki, T. Mikado and M. Chiwaki: A 600-MeV ETL electron storage ring, IEEE Trans. Nucl. Sci. NS, 30 (4II), 3133-3135 (1983).

[6] J. H. Hubbell and S. M. Seltzer: Tables of X-Ray Mass Attenuation Coefficients and Mass Energy-Absorption Coefficients from $1 \mathrm{keV}$ to $20 \mathrm{MeV}$ for Elements $\mathrm{Z}=1$ to 92 and 48 Additional Substances of Dosimetric Interest* (http:// www.nist.gov/pml/data/xraycoef/), accessed 2015-01-18.

[7] H. Toyokawa, S. Goko, S. Hohara, T. Kaihori, F. Kaneko, R. Kuroda, N. Oshima, M. Tanaka, M. Koike, A. Kinomura, H. Ogawa, N. Sei, R. Suzuki, T. Ohdaira, K. Yamada and H. Ohgaki: Recent progress in generation and application of AIST laser-Compton gamma-ray beam, Nucl. Instrum. and Methods in Phys. Res. A, 608 (1 Supp.), S41-43 (2009).

[8] H. Toyokawa, H. Ohgaki, T. Mikado and K. Yamada: High- energy photon radiography system using laserCompton scattering for inspection of bulk materials, Rev. Sci. Instrum., 73 (9), 3358-3362 (2002).

[9] H. Toyokawa: Gamma-ray CT using laser-Compton photon beam, Oyo Buturi, 78 (4), 351-354 (2009) (in Japanese).

[10] H. Toyokawa: Industrial imaging method using high-energy photon beam CT, Proc. 3rd International Workshop on
Process Tomography (IWPT-3), 95(1)-95(8) (2009).

[11] H. Toyokawa, H. Kanada, T. Kaihori, M. Koike and K. Yamada: Application of high-energy photon CT system with laser-Compton scattering to non-destructive test, IEEE Trans. Nucl. Sci., 55 (6), 3571-3578 (2008).

[12] Evaluation Subcommittee, Committee on Industrial Science and Technology, Industrial Structure Council: "Jisedai 3 Jigen Naigai Keisoku No Hyoka Kiban Gijutsu Kaihatsu" Jizen Hyoka Hokokusho (Preliminary Evaluation Report of the "Development of Core Evaluation Technology for Next-Generation 3 Dimensional Internal and External Measurement") (2012) (in Japanese).

[13] R. Suzuki: Development of battery-operated portable highenergy X-ray sources - Innovation in X-ray non-destructiveevaluation, Synthesiology, 2 (3), 237-243 (2009) (in Japanese) [Synthesiology English edition, 2 (3), 221-228].

[14] Japan Science and Technology Agency (2014-10-28): Press Release, JST Report, No. 1064, http://www.jst.go.jp/pr/info/ info1064/index.html, accessed 2015-01-18 (in Japanese).

\section{Author}

\section{Hiroyuki TOYOKAWA}

Completed the doctorate course at the Graduate School of Engineering, Nagoya University in 1997. Doctor (Engineering). Joined the Electrotechnical Laboratory, Agency of Industrial Science and Technology in 1997. Photonics Research Institute, AIST in 2001; Research Institute of Instrumentation Frontier (RIFF), AIST in 2001; Group Leader, Quantum

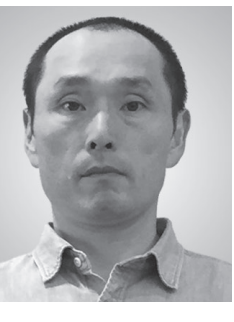
Radiation Research Group, RIFF-AIST in 2010; and Group Leader, Small Quantum Beam Source Group, RIFF-AIST since 2013.

\section{Discussions with Reviewers}

\section{Overall}

Comment (Naoto Kobayashi, Center for Research Strategy, Waseda University)

This paper describes the application of the laser Compton scattering technology to radiography. It organizes the abundant research results that the author has achieved over the years, and describes the process by which the technologies were selected and integrated to achieve the goal. This paper has solid content that is appropriate for Synthesiology.

However, the part on the LCS application in the first half of the paper is a review of the contents that were published in other papers, and does not necessarily address the synthesiological research that is particular to Synthesiology. As a paper of synthesiology, I think you should describe in order, what kind of R\&D scenario was created to realize the specific goal (application of LCS to radiography), how the elemental technologies were selected, and how they were integrated to realize the scenario. Also, I think you need to add a descriptive diagram to clarify your arguments.

\section{Main focus of the paper \\ Comment (Shingo Ichimura, Strategic Innovation Office, Nagoya University)}

As you described in the discussion, why don't you set the 
main focus of the paper on the following point: "Basic research results of science and technology conducted by the research institutes are required to be closely bound to corporate activities in many cases. This type of research is "bridging research" or translational research. Bridging research is work whereby one's concerns and thinking are organized and gradually shaped into a form that can be readily accepted by society." In other words, I think you should make the "methodology of bridging research" as the main focus of the paper.

By applying this methodology to the case of "high-energy photon beam generation technology and its application to nondestructive testing," you would be able to organize the contents described in the first half of the paper to show how specifically you sought the landing points of the following issues.

Technical level might be lowered if we easily compromised to produce the outcomes in a short time, or we may become isolated from society if we keep pursuing our own way.By applying this methodology to the case of "high-energy photon beam generation technology and its application to nondestructive testing," why don't you organize the contents described in the first half of the paper to show how specifically you sought the landing points of the following issues?

Technical level might be lowered if we easily compromised to produce the outcomes in a short time, or we may become isolated from society if we keep pursuing our own way.

\section{Comment (Naoto Kobayashi)}

In the abstract it is written "a summary of the development process (by which a technology takes one form) is outlined," and thinking that the "form" is "contribution to society" that is valuable for a Synthesiology paper, I think it is better to reorganize the paper with focus on "process" and "discussion." In that sense, if the "bridging research" is set as a goal, I suggest you structure the paper as follows: the contents in chapters 6 and 7 should be presented as a scenario to achieve the goal; the "process" of selection and integration of individual LCS technologies should be described as the elemental technologies that comprise the scenario; and the discussion should be provided in the final chapter.

\section{Answer (Hiroyuki Toyokawa)}

Taking your advice into consideration, I totally changed the structure of the paper. The review of technology was kept to a minimum, and the number of chapters was reduced to four.

Chapter 1 describes the background of research and the research disciplines that will be discussed in the paper. Chapter 2 explains the academic background and the process of research along with technological reviews. Chapter 3 presents the specific example of the process of selection and integration of technology. Also, I added Fig. 8 "Improvement of elemental technologies, elements that determine the performance index, and relationship of sophistication of devices and methods" that shows the overall goal and the structure of the technology to achieve the goal. Chapter 4 provides supplementary explanation to the facts presented in the previous chapter, and discusses the significance and positioning of the results. In this way, I attempted to expand this paper to the methodology of bridging research.

However, the task of raising the paper to the level of methodology of bridging research is quite difficult. If I try to universalize the research to a methodology, it tends to get summarized into general terms. The essence of the problem is that the granularity of various information shifts when moving from a specific case to a general discussion, and I think this is exactly the difficulty of this paper.

\section{Reorganization of the paper}

\section{Comment (Shingo Ichimura)}

In the revised paper, chapters 3 and 4 describe the contents according to the topics numbered as follows:

(1) Elemental technologies are developed (in this case five years; outside funds that allow basic research were used).

(2) After demonstrating the principle, use research is started. At the same time, the device utility is sophisticated.

(3) Use research is conducted smoothly.

(4) Points of future developments for devices and technologies are organized.

(5) Whether the research will be continued is determined considering the cost-effectiveness ratio and situations of the organization.

(6) Technological development is started at once after the decision to continue is made.

In the revised paper, overall (1) (2) became chapter 3, while

(3) (6) became chapter 4. However, in terms of content, isn't (1) $\sim(4)$ the first phase of development, and (5) and (6) the following phase built upon the first phase? If this perspective is correct, I think you should reorganize chapters 3 and 4 , and divide the topics to a chapter for (1) (4) (title for chapter 3 can be left as is) and a chapter for (5) and (6). I think it will be easier to understand if you add subtitles corresponding to (1) (6). Also, I think the readers will better understand if you provide some detailed explanation for the following points:

A) Even if you select the method called the first-generation CT as "development of elemental technology," there must have been some developed item that was highly original of the author. One will understand better if there is a specific description on such an item. It can be presented in a simple diagram.

B) In the places that correspond to "sophistication of utility," what was the subject of investigation, what was lacking, and what advancement did you make to solve the issue? I think you should give a more detailed explanation.

C) In the essence part of the description for (5), I think you should add a bit more description on what decisions you made under what requirements (considerations), and how that led to the conclusion described. This will be useful in understanding the methodology of bridging research.

Answer (Hiroyuki Toyokawa)

I adjusted the whole structure of the paper based on your comments. Also, I have added, deleted, and moved explanations where appropriate. The details are provided in (1), (2), and (3) of chapter 3 . 